\title{
Stroke-Like Episodes Associated with Coronavirus Disease 2019 in a Child with MELAS Syndrome
}

\author{
Woo-Jin Kim, MD, Mi-Sun Yum, MD, Min-Jee Kim, MD, Han-Na Jang, MD, Tae-Sung Ko, MD \\ Department of Pediatrics, Asan Medical Center Children's Hospital, University of Ulsan College of Medicine, Seoul, Korea
}

Received: June 5, 2021

Revised: June 21, 2021

Accepted: June 22, 2021

\section{Corresponding author:}

Mi-Sun Yum, MD

Department of Pediatrics, Asan

Medical Center Children's Hospital, University of Ulsan College of Medicine, 88 Olympic-ro 43-gil, Songpa-gu, Seoul 05505, Korea Tel: +82-2-3010-3386

Fax: +82-2-473-3725

E-mail:misun.yum@gmail.com
The patient described in this report was the second child of healthy, non-consanguineous Korean parents. At 11 years of age, she was admitted to our clinic with fever, headache, and hemiparesis on the left side. On a neurologic examination, she showed facial palsy and decreased motor power (grade III) on the left side. Laboratory tests revealed elevated lactic acid $(3.3 \mathrm{mmol} / \mathrm{L})$. Brain magnetic resonance imaging (MRI) and angiography showed diffuse cortical and subcortical T2/fluid-attenuated inversion recovery (FLAIR) high signal intensity with gyral swelling in the right temporo-parieto-occipital lobe without steno-occlusive lesions (Supplementary Fig. 1). Mitochondrial DNA sequencing revealed m.3243A $>$ G heteroplasmy of the mitochondrially encoded TRNA-Leu (UUA/G)1 (MT-TL1) gene, and her condition was diagnosed as mitochondrial encephalopathy, lactic acidosis and stroke-like episode (MELAS) syndrome (Supplementary Fig. 1). She presented with severe sensorineural hearing loss (SNHL) on pure-tone audiometry and a decreased full-scale intelligence quotient of 52 based on an intelligence test. The ophthalmologic examination and echocardiography were unremarkable. We administered L-carnitine, pyridoxine, thiamine, decaquinon, and multivitamins, and she had no additional stroke-like episodes or neurologic deficit for 4 years. At 15 years of age, she visited our clinic be- cause of headache, difficulty understanding words, and a slow response to questions. She had a history of coronavirus disease 2019 (COVID-19) 3 weeks previously without definite pneumonia or respiratory difficulty. Laboratory test results, including COVID-19 polymerase chain reaction, were unremarkable, except for the presence of lactic acidosis (pH 7.242, lactic acid $3.0 \mathrm{mmol} / \mathrm{L}$ ). Chest radiography and echocardiography were also normal. Brain MRI revealed diffuse gyral swelling and high signal intensity on T2/FLAIR in the bilateral frontal and left temporal lobes and basal ganglia with restricted diffusion, consistent with new stroke-like lesions associated with MELAS syndrome (Fig. 1). After administration of high-dose arginine therapy, she was discharged on admission day 6 with improved verbal responses. One week later, she was readmitted to our medical center because of dysarthria, poor verbal response, and general weakness. Repeated brain MRI showed increased extent of the previous lesions (Supplementary Fig. 2). During hospitalization, lip twitching was observed, and electroencephalography showed diffuse suppressed background activity with intermittent delta slow waves over the left frontal areas (Supplementary Fig. 2). After conservative therapy, including arginine and gabapentin, her dysarthria and lip twitching gradually disappeared. On hospitalization day 12 , she returned to her usual condition

Copyright (C) 2021 Korean Child Neurology Society

This is an Open Access article distributed under the terms of the Creative Commons Attribution Non-Commercial License (http://creativecommons.org/licenses/by-nc/4.0/) which permits unrestricted non-commercial use, distribution, and reproduction in any medium, provided the original work is properly cited. 


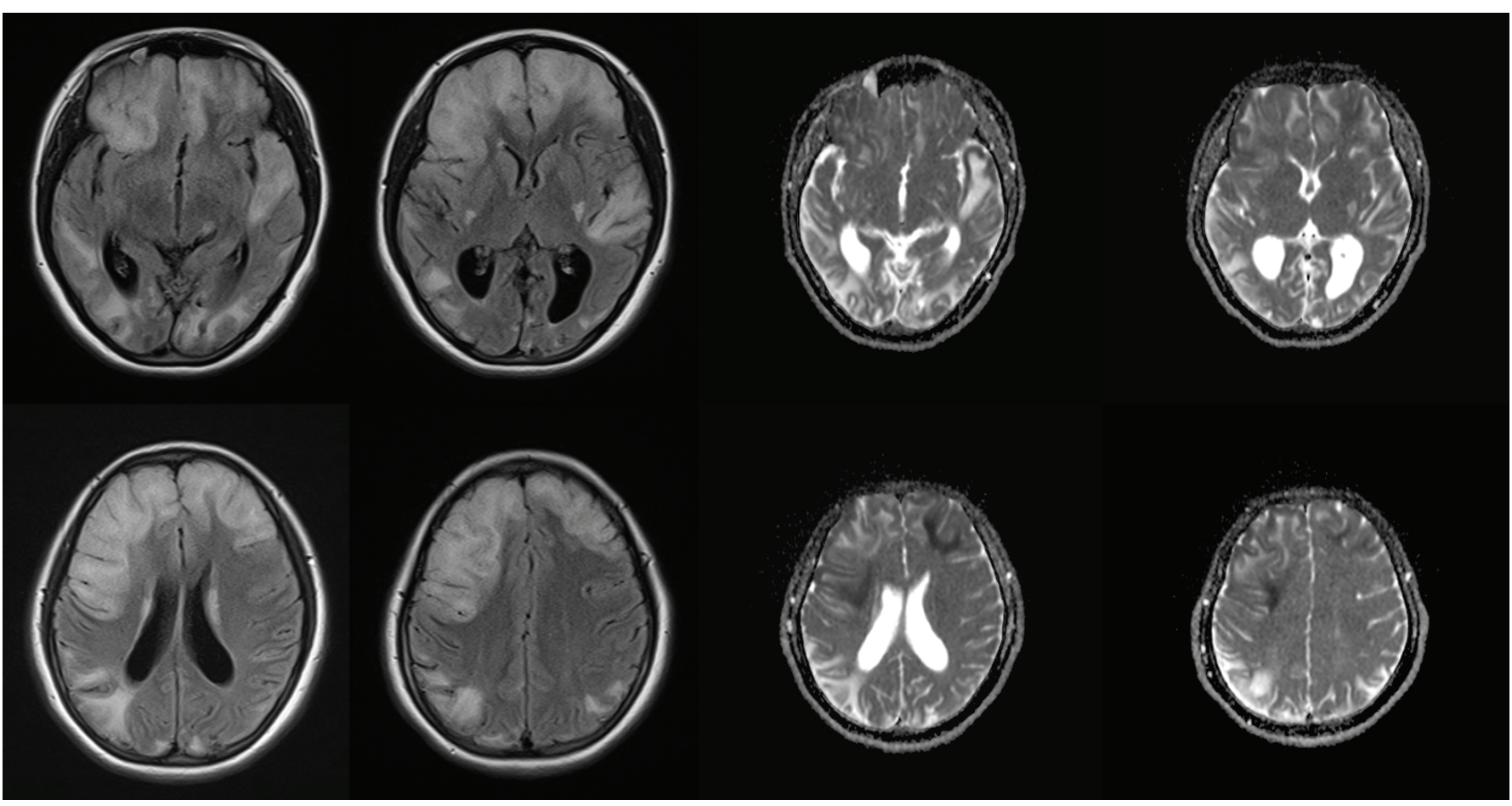

Fig. 1. Brain magnetic resonance imaging of first admission at 15 years of age.

and was discharged.

Here, we present a case of new stroke-like episodes after COVID-19 in a patient with MELAS syndrome. This case provides additional information regarding COVID-19 and could guide the evaluation and management of patients with mitochondrial disease during the current pandemic situation. Since the initial outbreak of severe acute respiratory syndrome coronavirus 2 (SARS-CoV-2), previously referred to as "the novel coronavirus," in December 2019, there is mounting evidence that COVID-19 causes not only respiratory illness but also a hypercoagulable state, leading to thrombotic complications including ischemic stroke [1]. In a recent review by the World Stroke Organization, comprising stroke experts from 18 countries, the risk of ischemic stroke in hospitalized patients with COVID-19 was approximately $5 \%$, and the median duration from the first symptoms of COVID-19 to stroke was 10 days [2]. Suggested mechanisms of the development of ischemic stroke in COVID-19 include vasculitis, a hypercoagulable state, and cardiomyopathy [1]. Additionally, Saleh et al. [3] suggested that mitochondrial dysfunction could occur in COVID-19. Stroke-like episodes are a cardinal feature of MELAS syndrome, occurring in $84 \%$ to $99 \%$ of patients along with seizure and lactic acidemia [4]. As in our patient, the most common cause of MELAS syndrome is a missense mitochondrial DNA mutation (m.3243A > G) in the MT-TL1 gene, encoding mitochondrial transfer RNA for leucine; this mutation reduces mitochondrial protein synthesis and energy production [5]. Energy deficiency stimulates the proliferation of abnormal mitochondria in smooth muscles and small blood vessel endothelial cells, causing angiopathy, cellular dysfunction, and microvasculature perfusion disorders, leading to segmental vasodilation defects and subsequent vasogenic edema, which are thought to contribute to the occurrence of stroke-like episodes $[6,7]$. The drug, chickenpox infection, and fever have been reported as potential triggering factors of stroke-like episodes [8]. Our patient with MELAS syndrome was relatively stable, without stroke-like episodes, and was doing well, except for SNHL and mild intellectual disability. However, the stroke-like episodes recurred after COVID-19. It is difficult to identify the exact pathomechanism, although one possibility is that the COVID-19 infection collapsed the compensatory state of preexisting mitochondrial dysfunction, energy failure, neuronal cytopathy, and angiopathy, and then the inflammatory response of vascular endothelium and additional mitochondrial dysfunction led to the strokelike episode in our patient. Although there are accumulating reports of acute cerebrovascular disease as a complication of COVID-19, the development of stroke during COVID-19 is more frequent in patients with vascular risk factors, including diabetes mellitus (odds ratio [OR], 5.56; 95\% confidence interval [CI], 3.34 to 9.24 ), hypertension (OR, 7.35; 95\% CI, 1.94 to 27.87 ) and coronary artery disease (OR, 3.12; 95\% CI, 1.61 to 6.02$)$ [9]. We need to raise awareness of mitochondrial encephalopathy as a risk 
factor for stroke in COVID-19 and provide appropriate guidelines to these patients.

\section{Supplementary materials}

Supplementary materials related to this article can be found online at https://doi.org/10.26815/acn.2021.00395.

\section{Conflicts of interest}

No potential conflict of interest relevant to this article was reported.

\section{ORCID}

Woo-Jin Kim, https://orcid.org/0000-0002-2608-579X

Mi-Sun Yum, https://orcid.org/0000-0002-5986-5258

\section{Author contribution}

Conceptualization: MSY and TSK. Data curation: WJK and HNJ. Formal analysis: WJK, MJK, and HNJ. Funding acquisition: WJK. Methodology: MJK. Project administration: WJK. Visualization: WJK and MJK. Writing-original draft: WJK. Writing-review \& editing: MSY, MJK, and TSK.

\section{References}

1. Spence JD, de Freitas GR, Pettigrew LC, Ay H, Liebeskind DS, Kase CS, et al. Mechanisms of stroke in COVID-19. Cerebro- vasc Dis 2020;49:451-8.

2. Qureshi AI, Abd-Allah F, Al-Senani F, Aytac E, Borhani-Haghighi A, Ciccone A, et al. Management of acute ischemic stroke in patients with COVID-19 infection: insights from an international panel. Am J Emerg Med 2020 38:1548.e5-7.

3. Saleh J, Peyssonnaux C, Singh KK, Edeas M. Mitochondria and microbiota dysfunction in COVID-19 pathogenesis. Mitochondrion 2020;54:1-7.

4. El-Hattab AW, Adesina AM, Jones J, Scaglia F. MELAS syndrome: clinical manifestations, pathogenesis, and treatment options. Mol Genet Metab 2015; 116:4-12.

5. Goto Y, Nonaka I, Horai S. A mutation in the tRNA(Leu) (UUR) gene associated with the MELAS subgroup of mitochondrial encephalomyopathies. Nature 1990;348:651-3.

6. Hasegawa H, Matsuoka T, Goto Y, Nonaka I. Strongly succinate dehydrogenase-reactive blood vessels in muscles from patients with mitochondrial myopathy, encephalopathy, lactic acidosis, and stroke-like episodes. Ann Neurol 1991;29:601-5.

7. Goto Y, Horai S, Matsuoka T, Koga Y, Nihei K, Kobayashi M, et al. Mitochondrial myopathy, encephalopathy, lactic acidosis, and stroke-like episodes (MELAS): a correlative study of the clinical features and mitochondrial DNA mutation. Neurology 1992;42(3 Pt 1):545-50.

8. Koga Y, Povalko N, Nishioka J, Katayama K, Kakimoto N, Matsuishi T. MELAS and L-arginine therapy: pathophysiology of stroke-like episodes. Ann N Y Acad Sci 2010;1201:104-10.

9. Nannoni S, de Groot R, Bell S, Markus HS. Stroke in COVID-19: a systematic review and meta-analysis. Int J Stroke 2021;16:13749. 